Recepción: 13 / 09 / 2018

Aceptación: 17 / 11 / 2018

\title{
Emprendimiento social en Ecuador: análisis del enfoque social vs. Enfoque de mercado
}

\section{Social entrepreneurship in Ecuador: analysis of the social approach and. market approach}

\section{Empreendedorismo social no Equador: análise da abordagem social e. abordagem de mercado}

\author{
Danilo J. Altamirano-Analuisa ${ }^{\mathrm{I}}$ \\ danilojaltamirano@uta.edu.ec \\ Ruth A. Zamora-Sánchez II \\ ra.zamora@uta.edu.ec \\ Marcelo J. Mancheno-Saá III \\ mj.mancheno@uta.edu.ec
}

Correspondencia: danilojaltamirano@uta.edu.ec

\footnotetext{
I Magíster en Gerencia Empresarial MBA Mención Gestión de Proyectos, Doctor Dentro del Programa Oficial de Doctorado en Desarrollo Local y Economía Social, Máster Universitario en Economía y Desarrollo Territorial, Ingeniero En Finanzas, Docente de la Universidad Técnica de Ambato, Ecuador.

II Máster Universitario en Dirección Empresarial Desde la Innovación y la Internacionalización, Licenciada en Administración y Dirección de Empresas, Diplomada en Ciencias Empresariales, Docente de la Universidad Técnica de Ambato, Ecuador.

III Magíster en Administración de Empresas Mención Planeación, Máster Universitario en Marketing Digital y Comercio Electrónico, Ingeniero Comercial con Mención en Marketing, Docente de la Universidad Técnica de Ambato, Ecuador.
} 


\section{Resumen}

La construcción de un sistema económico social y solidario requiere de modelos organizacionales generadores de valor social y económico hacia la transformación de sociedades dinámicas. Los patrones culturales, sociales y económicos basados en principios de cooperación y solidaridad instauran sociedades más igualitarias y con justicia social. En tal virtud, este estudio de revisión teórica y de tipo descriptivo genera un análisis comparativo entre el enfoque social y de mercado desde una perspectiva dinámica de los modelos de desarrollo y transformación socioeconómica, considerando marcos conceptuales y teóricos, y su aplicación en el ecosistema emprendedor, a fin de contribuir con la sociedad de propietarios, productores y emprendedores. El estudio evidencia similitudes y diferencias entre el emprendimiento social y de mercado en su naturaleza y sus fines.

Palabras clave: emprendimiento social; valor; mercado; desarrollo socioeconómico; cambio social.

\section{Abstract}

The construction of a social and solidarity economic system requires organizational models generating social and economic value towards the transformation of dynamic societies. Cultural, social and economic patterns based on principles of cooperation and solidarity establish more egalitarian societies and social justice. Thus, this study of theoretical and descriptive review generates a comparative analysis between the social and market approach from a dynamic perspective of the models of development and socioeconomic transformation, considering conceptual and theoretical frameworks, and their application in the entrepreneurial ecosystem, in order to contribute to the society of owners, producers and entrepreneurs. The study shows similarities and differences between the social and market entrepreneurship in its nature and its aims.

Keywords: Social entrepreneurship; value; market; socioeconomic development; social change.

\section{Resumo}

A construção de um sistema social e solidário de modelos organizacionais de valor social e econômico tem a transformação de sociedades dinâmicas. Los patrones culturales, sociais y tempos basados en principios de cooperación y solidaridad instauran sociedades más igualitarias 
y con justicia social. Estas virtudes, este é um método de análise e descrição descritiva da geração de uma análise comparativa entre o enfoque social e de mercado de uma perspectiva dinâmica dos modelos de desenvolvimento e de transformação socioeconômica, considerando marcos conceituais e teóricos, e sua aplicação no ecossistema emprendedor, a fin de contribuir com a sociedade de propietarios, productores e empreendedores. El estudio evidencia similitudes y diferencias entre o empreendedorismo social e de mercado em su natureza y sus multas.

Palavras chave: Emprestimiento social, valor, mercado, socioeconómico, cambio social.

\section{Introducción}

El emprendimiento ha sido arduamente estudiado y considerado un factor clave de esperanza para los gobiernos y agentes sociales, debido a su contribución al desarrollo de los países, siendo necesario la participación de entidades públicas y privadas, quienes apoyen la creación de empresas a través de diversos programas, tales como el desarrollo de unidades, aceleradores de emprendedores, redes de cooperación, incubadoras, entre otras (Salinas \& Osorio, 2012).

El término de emprendimiento social se adopta fuertemente a partir del siglo XXI para designar formas de organización novedosas, también denominadas modelo híbrido que resuelven problemas sociales, es decir, que se trata de un modelo de empresa más humano y sustentable, relacionada con el sector de la economía social. La empresa social al combinar rentabilidad y misión social ofrece la posibilidad de resolver problemas sociales y no perecer ante un capitalismo capaz que no permite la sobrevivencia a largo plazo de los que sólo se concentran en el bien común (Mukesh, VanSandt \& Baugous, 2009).

Alemán y Coronado (2014) determinan al emprendimiento social como aquel que está orientado a la inclusión social de los grupos más vulnerables a la dinámica económica de los países, a la vez que se genere valor social, favoreciendo oportunidades para la transformación social. Mientras que Santos, Barroso y Guzmán (2013) manifiestan que dicho emprendimiento social debe potenciar el establecimiento de una estructura económica mundial más equitativo.

De las definiciones anteriores, se puede resumir que el emprendimiento social, como mecanismo de mejora social, conlleva una transformación integral dinamizadora de la economía, cuyo objetivo es la creación de valor social, que se traduce en dar solución a problemas de índole social dentro de un determinado entorno; como valor económico, que implica una mejor 
asignación de los recursos (Dacin \& Dacin, 2011). No obstante, Santos (2012: 337) considera más apropiado reparar en un concepto de valor más global "definido en términos del aumento en la utilidad de los miembros de la sociedad".

El alcance de las actividades económicas de quienes pertenecen al sector de la economía popular supone un aporte para la construcción de un sistema económico social y solidario, el cual beneficia a los países; por ello, la empresa resultante del emprendimiento social es considerada como un modelo organizacional innovador, eficaz sostenible y creadora de valor (PalaciosNúñez, 2010).

Para que las nuevas organizaciones puedan operar es necesario contar con un marco legal (micro - macro económico) que estimule a las empresas; esto es, crear políticas públicas que favorezcan las actividades emprendedoras, su reconocimiento, financiación y apertura de mercados, como se aprecia en el apoyo a la dinámica empresarial en regiones y países emergentes como India, Sureste Asiática, Irlanda o Europa del Este (Kantis, Ishida \& Komori, 2002; Minniti, Bygrave \& Autio, 2006; Acs y Amorós, 2008). En el caso de los países de América Latina, la creación de un ecosistema innovación y de emprendimiento social se ha visto principalmente limitado debido al lento e inestable crecimiento de sus economías y escasez de recursos financieros dirigidos a la generación de actividades vinculadas con el emprendimiento (Fonseca, Rueda \& García, 2015).

Aunque los patrones de emprendimiento dependen del desarrollo socioeconómico de los países y su alcance a las nuevas innovaciones tecnológicas (Ramírez, Bernal, \& Fuentes, 2013), los resultados proporcionados por el Report Global Entrepreneurship Monitor (GEM, 2017) entre los años 2016-2017, evidencian que la acción emprendedora de Ecuador respecto a la Actividad Temprana de Emprendimiento (TEA) se encuentra en la segunda posición de entre 65 países, esto es, que, aunque las acciones de emprendimiento se encuentran aún en un estado incipiente, las motivaciones y ambiciones de los empresarios y agentes sociales van reforzando una cultura emprendedora en el país (Padilla-Martínez, Quispe-Otacoma, Nogueira-Rivera \& HernándezNariño, 2016).

Esta reciente realidad social está vinculada con el auge de una economía social, la cual aparece como un espacio de acción no sólo conformado por un único sujeto, sino también por colectivos, familias y comunidades, que, basado en valores de cooperación y solidaridad, satisfacen 
necesidades de la sociedad mediante la aplicación de mecanismos de organización económica ajenos al sistema capitalista global (Coragio, 2011).

La Constitución de la República de Ecuador en 2008 establece las bases para instaurar una economía social y solidaria en donde se consideran los principios de reciprocidad, cooperación solidaria, inclusión y justicia social, en pro a solventar las demandas de la sociedad ecuatoriana. Sin embargo, es indispensable que el gobierno promueva transformaciones impostergables, encaminadas a garantizar la producción y reproducción de las condiciones materiales e inmateriales que propicia el Buen Vivir (Sumak Kawsay) y la satisfacción de las necesidades vitales de la sociedad (Coragio, 2009).

Una manera de contribuir a ese cambio es la medida implementada por el Código Orgánico de la Producción, Comercio e Inversiones, que estable necesario "generar un sistema integral para la innovación y el emprendimiento, para que la ciencia y tecnología potencien el cambio de la matriz productiva; y para contribuir a la construcción de una sociedad de propietarios, productores y emprendedores" (Código Orgánico de la Producción, Comercio e Inversiones, 2010:5).

El Código expone la importancia que tiene la capacitación y desarrollo tecnológico para construir zonas equipotentes que potencien sus ventajas comparativas y competitivas, donde las instituciones de educación superior son las llamadas a implementar articuladamente un plan de economía social de los conocimientos, creatividad e innovación.

Por todo lo mencionado anteriormente, se considera de interés llevar a cabo la presente investigación, que tiene por propósito generar un análisis comparativo entre los enfoques social y de mercado desde una perspectiva dinámica de los modelos de desarrollo y transformación socioeconómica, considerando marcos conceptuales y teóricos, y su aplicación en el ecosistema emprendedor.

\section{Desarrollo}

En las últimas décadas, el emprendimiento social se ha constituido en un proceso socioeconómico donde el sujeto social constituye el factor clave por cuanto genera valor en la 
perspectiva de crear transformación social sustentada en innovación social, dando lugar a nuevos modelos de desarrollo (Seelos \& Mair, 2005).

Aunque se han realizado estudios sobre emprendimiento vinculados al proceso de crecimiento económico, no siempre se ha dado un papel central en el análisis económico que ha estado dominado por teorías del proceso de producción, donde la acumulación mecánica de capital y trabajo ha sido su misión, más no la visualización de oportunidades para promover igualdad de oportunidades y la reducción de la pobreza.

Alemán y Coronado (2014) manifiestan que el emprendimiento social se diferencia de otro tipo de emprendimiento, porque éste está orientado a la inclusión social de los grupos más indefensos a la dinámica económica de los países, a la vez que busca crear valor social, y fomentar el cambio social. Esta nueva actividad puede ser concebida como un emprendimiento con misión social (Martin \& Osberg, 2007), una actividad empresarial que tiene por finalidad un sistema social (Austin Stevenson \& Wei-Skillern, 2006), la búsqueda de un nuevo ambiente económico, social y ambiente de iniciativa empresarial (Haugh, 2007) o "una práctica social, susceptible de entenderse según la expresión de la conducta humana" (Orrego, 2009:4).

En este caso, es importante considerar a la economía y al emprendimiento como parte de una evolución de construcción social en el que la organización de la producción, distribución, comercialización y consumo de bienes y servicios se orienta a la solución de las necesidades y deseos legítimos de las comunidades (Hodgson, 2017) y no simplemente para la acumulación de riqueza (Jácome, 2016). Esto es, que el emprendimiento es actividad compleja, multifacético y las definiciones simples no dan cuenta de la heterogeneidad de las acciones innovadoras que este fenómeno abarca, dado su naturaleza social e identidad cultural.

La construcción de una economía popular y solidaria requiere, por su alcance y naturaleza, evitar situaciones de vulnerabilidad que pueden afectar negativamente la sobrevivencia de los emprendimientos y de sus actores. El dinamismo socio económico implícito en la acción de emprender demanda anticipación y congruencia entre las acciones individuales y colectivas de las organizaciones sociales y de las diversas instancias estatales, con momentos de planificación, evaluación y reflexión conjunta sobre los procesos puestos en marcha (MIES, 2009). 
La trascendencia de las actividades económicas de quienes pertenecen al sector de la economía popular implica una contribución de los cimientos de un sistema económico social y solidario, el cual beneficia a los países; por ese motivo, la organización resultado del emprendimiento social es apreciada como un modelo organizacional innovador, eficaz, sostenible y creadora de valor (Palacios-Núñez, 2010).

En Ecuador, el sector de la economía social y solidaria se vio beneficiada por el crédito de desarrollo humano, que ha constituido un incentivo para los emprendimientos productivos populares otorgando microcréditos para la creación de negocios. Aunque el crédito ha sufrido diversos cambios desde la década de los noventa, continúan existiendo algunas debilidades como la normativa jurídica respecto a la protección social, la relación entre el uso del crédito y la mejora de las condiciones del beneficiario o los requisitos exigidos para otorgarlo (Bermeo, 2013).

Hay que considerar que la economía solidaria tiene sus cimientos en el valor social, la cooperación, solidaridad y reciprocidad, en donde los individuos se ayudan unos a otros para dar solución a problemas de la comunidad y mejorar las condiciones de los grupos más vulnerables. Estas acciones deben estar en armonía con el marco político, social y económico vigente en el país (Molina \& Córdova, 2014).

De acuerdo con esto, Bargsted (2013) detalla que el objetivo principal del emprendimiento es crear valor social en un grupo de individuos por medio de actividades empresariales o socialcomunitarias. En el contexto del emprendimiento social será una alternativa económica que genere el cambio, que busca resolver problemas de la sociedad de una manera inclusiva y eficiente, reconociéndose la naturaleza compleja y heterogénea de su impacto.

Chell (2007) manifiesta que todo emprendimiento contiene un equilibrio entre comportamiento social y económico, los mismo que originan valor social y valor económico; dicho valor supone la base para diferenciar un emprendimiento de índole social de un emprendimiento de negocios.

El concepto de valor es determinante en los principios básicos de nuestras acciones por cuanto nos permite compartir normas e identificar el comportamiento deseado y adecuado en las diferentes situaciones de interacción social. Los valores tienen una influencia en la percepción e interpretación de las situaciones, ya que, si son activados, ejercen una influencia en el 
comportamiento, lo cual induce en el poder atractivo sobre las acciones, ya que estimula la planificación de la acción (Schwartz, 2005). La siguiente tabla detalla los valores desde distintas dimensiones (Tabla 1):

Tabla 1. Valores individualistas y colectivistas

\begin{tabular}{|llc|}
\hline Dimensiones & Tipo de valor & Valores especificos \\
\hline Individualismo & Poder & Poder Social, Autoridad,Riqueza \\
& Logro & Exitoso, Capaz, Ambicioso \\
& Hedonismo & Placer, Disfrutar de la Vida \\
& Estimulación & Vida Variada \\
& Auto Dirección & Creatividad, Libertad \\
Colectivismo & Benevolencia & Ayudar, Perdonar, Honestidad \\
& Tradición & Humilde, Devoto, Moderado \\
& Conformidad & Obediencia, Buenos Modales \\
Mixtos & Universalismo & Tolerancia, Justicia Social, Igualdad \\
& Seguridad & Orden Social, Seguridad \\
\hline
\end{tabular}

Fuente: Schwartz (2005).

\section{Elaboración: propio.}

El valor social debe entenderse como aquel que generan los emprendimientos sociales es el servicio o producto de buscar dar solución a problemas sociales y promover la inclusión de los grupos más vulnerables, lo cual contribuye a mejorar el desarrollo socioeconómico de los países (Salinas \& Osorio, 2012); mientras que, el valor económico será el que proviene de empresas, sociales o no, que tienen por finalidad obtener beneficios económicos, a la vez, que aspiran a ser más competitivas en el mercado local o global (Dacin \& Dacin, 2011). Es decir, que existen empresas sociales lucrativas y empresas sociales no lucrativas, diferenciándose tanto en el valor como en la misión de la organización (Moreira \& Urrilagoitia, 2011).

En tal sentido, hablar de valor social y valor económico del emprendimiento social incluye reconocer su naturaleza interdisciplinaria y complejidad. De igual manera, expone la necesidad de diseminar un paquete de innovaciones, información y recursos técnicos que pueden reconfigurarse de manera que puedan ser adaptados y utilizados por grupos marginales; y finalmente, la construcción de un movimiento de alianzas con organismos públicos y privados que ayuden a dar solución a problemas más graves de la sociedad (Alvord, Brown, \& Letts, 2004). 
Esta diferencia en el valor resultante del emprendimiento da lugar a dos enfoques: el socialasistencial y el económico, que, aunque ambos contribuyen al desarrollo socioeconómico de los países mediante la disminución de la pobreza, la generación de empleo o la mejora de los sistemas productivos, se distinguen en que el enfoque social persigue dar solución a cuestiones referentes a salud, educación, vivienda o seguridad, sin perseguir un beneficio económico; en cambio, el enfoque económico, además de contribuir al desarrollo económico mediante la generación de empleo, busca obtener rendimientos empresariales (Alburquerque-Llorens, 2004).

Bajo esta perspectiva, el progreso de los procedimientos de democratización y descentralización ha forzado a los hacedores de las políticas a buscar planteamientos más adecuados a las dificultades y actuales demandas de la sociedad (Affonso, 2000). En el caso de Latinoamérica, el establecimiento de un ambiente innovador y de emprendimiento social ha estado limitado por un pausado e inestable crecimiento de sus economías e insuficiencia de recursos financieros orientados a la generación de actividades relacionadas con el emprendimiento (Fonseca, Rueda \& García, 2015).

En Ecuador, los organismos y su estructura legal que contribuye a la economía popular y solidaria son: la Constitución de la República del Ecuador de 2008, el Plan Nacional de Desarrollo 2017-2021 denominado Plan Toda una Vida, el Plan Nacional de Desarrollo para el Buen Vivir 2013-2017, el Instituto Nacional de Economía Popular y Solidaria (IEPS), y la Ley Orgánica de la Economía Popular y Solidaria del Sector Financiero, Popular y Solidario (LOEPS). Todos ellos, son los encargados de otorgar orientación a las distintas formar de organización social como las cooperativas, asociaciones, comunidades y las unidades económicas populares en el país (Molina \& Córdova,2014).

Dada la complejidad del emprendimiento social, a continuación, se muestra las dimensiones de la acción emprendedora desde un enfoque social y otro de mercado (Tabla 2):

Tabla 2. Dimensiones emprendedoras: enfoque social y enfoque de mercado

\begin{tabular}{|ccc|}
\hline DIMENSIÓN & ENFOQUE SOCIAL & ENFOQUE DE MERCADO \\
\hline PRINCIPIOS & $\begin{array}{l}\text { Inclusión, justicia social, } \\
\text { cooperación, solidaridad, igualdad. }\end{array}$ & Libertad, equidad, seguridad. \\
\hline
\end{tabular}




\begin{tabular}{lll} 
Economía social. & Economía de mercado. \\
& Valor social. & Valor económico. \\
& Emprendimiento social. & Emprendimiento empresarial. \\
& Tejido social. & Tejido empresarial. \\
NATURALEZA & Estructura social. & Estructura empresarial. \\
& Cultura participativa. & Cultura individualista. \\
& Dinámica social. & Dinámica empresarial. \\
& Grupos vulnerables. & Grupos económicos. \\
& Demandas sociales. & Demandas de mercado. \\
& & \\
Transformación social. & Transformación socio económica. \\
Progreso social. & Progreso económico. \\
Desarrollo social. & Desarrollo económico. \\
Generación de empleo. & Generación de empleo. \\
Generación de oportunidades. & Generación de oportunidades. \\
Creación de empresas. & Creación de empresas. \\
Innovación social. & Innovación empresarial. \\
\hline FINES & \\
\hline
\end{tabular}

\section{Elaboración: propia}

En la tabla 2 se presentan unas características dimensionales manifestadas en enfoques de orden social y de mercado. El enfoque social tendrá lugar en el desarrollo de una economía social, en donde rigen los principios de cooperación, inclusión, igualdad y solidaridad. En este enfoque, las actividades de emprendimiento social buscan generar valor bajo el enfoque de crear un cambio y transformación social sustentada en innovación social (Seelos \& Mair, 2005), a la vez, que se espera que dicho emprendimiento genere oportunidades de empleo y progreso social, dando lugar un nuevo modelo de desarrollo alternativo.

El enfoque de mercado considera aquellos aspectos que tienen lugar en la economía de mercado, en donde la competitividad de las organizaciones, las exigencias productivas, las continuas 
innovaciones de productos, el beneficio económico y satisfacer las demandas de los clientes, posee una importancia mayor a obtener sólo un valor social. Las empresas que conforman una economía de mercado aprovechan las oportunidades del tejido empresarial para impulsar el desarrollo económico de los países (Acs y Amorós, 2008), esto es, que el sistema productivo existente permite a los agentes emplear con criterios de eficiencia los recursos disponibles a fin de mejorar los niveles de competitividad y productividad (Fernández, Narváez \& Senior, 2008).

Si bien cada enfoque tiene un objetivo distinto, ambos dependen de una coordinación política y social para contribuir al desarrollo económico de los países, alineados a mejorar las condiciones de la sociedad, a la vez, que se persigue la obtención de beneficios económicos, es decir, generar un crecimiento económico basado en la equidad, la justicia social y una economía sostenible que eleve la calidad de vida y bienestar de sus pobladores (Casanova, 2004), y en donde ambos enfoques contribuyan al desarrollo sustentable.

\section{Conclusiones}

La heterogeneidad de la economía social y solidaria demanda una base teórica y conceptual interdisciplinaria para interpretar la dimensión de los emprendimientos sociales. Dada su naturaleza y finalidad se manifiesta como modelo de desarrollo alternativo, por cuanto pretende armonizar y complementar el valor social y económico de los emprendimientos basados en principios de la cooperación, solidaridad y reciprocidad.

Esta economía alternativa se destaca que combinar rentabilidad y misión social, ofreciendo la posibilidad de resolver problemas sociales y mejorar las condiciones socioeconómicas de los grupos vulnerables en sus acciones individuales y colectivas. En el caso de Ecuador, la economía social y solidaria se ha visto beneficiada por disponer de marco legal e institucional que contribuyen a un proceso de construcción social, que respaldan las iniciativas de emprendimiento social.

Lo antes expuesto, origina un enfoque de índole social en el que rigen los principios de la economía solidaria, y que se diferencia del modelo tradicional de mercado al no perseguir como objetivo principal el lucro económico. No obstante, tanto las organizaciones con enfoque social como de mercado promueven el dinamismo de una sociedad impulsada por el empleo de calidad, el aprovechamiento de oportunidades y contribución al desarrollo económico. 
Sin embargo, todavía es necesario actualizar las políticas públicas que favorezcan las actividades emprendedoras, a la vez, que estimule la creatividad e innovación en el país.

\section{Referencias Bibliográficas}

Acs, Z. J., \& Amorós, J.E. (2008). Entrepreneurship and competitiveness dynamics in Latin America. Small Business Economics, 31(3), 305-322.

Alburquerque Llorens, F. (2004). Desarrollo económico local y descentralización en América Latina. Revista de la CEPAL, (82), 157-171.

Alemán, F., \& Coronado, C. (2014). Emprendimiento social innovador: El caso BIORED Colombia SAS en Antioquia Colombia. Institute for Business \& Finance Research. En Global Conference on Business and Finance Proceedings, 9(2), p. 508.

Alvord, S., Brown, L., \& Letts, C. (2004). Social entrepreneurship and socieral tranformation: An exploratory study. The Journal of Applied Behavioral Science, 40(3), 260-282.

Austin, J., Stevenson, H., \& Wei-Skillern, J. (2006). Social and commercial entrepreneurship: Same, different, or both? Entrepreneurship: Theory \& Practice, 30(1), 1-22.

Bargsted, A. M. (2013). El emprendimiento social desde una mirada psicosocial. Civilizar Ciencias Sociales y Humanas, 13(25), 121-132.

Bermeo, F. (2013). De las transferencias monetarias al sistema nacional de inclusión y equidad social. en: Hacia una reforma del bono de desarrollo humano. Quito: Ediciones Abya-Yala.

Casanova, F. (2004). Desarrollo local, tejidos productivos y formación. Abordajes Alternativos para la Formación y el Trabajo de los Jóvenes. Oficina Internacional del Trabajo-OITCINTERFOR, Montevideo.

Chell, E. (2007). Social enterprise and entrepreneurship: towards a convergent theory of the entrepreneurial process. International small business journal, 25(1), 5-26.

Código Orgánico de la Producción, Comercio e Inversiones (2010). Obtenido en red en el enlace: file://C:/Users/USER/AppData/Local/Temp/COPCI.pdf 
Coragio, J. (2009). Marco conceptual y lineamiento de acción para el Instituto de Economía Popular y Solidaria. Quito.

Coraggio, J. (2011). Economía social y solidaria. El trabajo antes que el capital. Quito: Abya Yala.

Dacin, M. T., \& Dacin, P. A. (2011). Social entrepreneurship: A critique and future directions. Organization Science, 22(5), 1203-1213.

Fernández, G., Narváez, M., \& Senior, A. (2008). El desarrollo local sobre la base de la asociatividad empresarial: una propuesta estratégica. Opción, 24(57), 74-92.

Fonseca, M., Rueda, C., \& García, H. (2015). Comportamiento económico, social, político e histórico de la actividad empresarial (emprendimiento) en las provincias de Cotopaxi, Tungurahua, y Pastaza del Ecuador. Yachana Revista Científica, 4, 54-64.

GEM. (2017). Report Globla Estrepreneurship Monitor. London.

Haugh, H. (2007). New strategies for a sustainable society: The growing contribution of social entrepreneurship. Business Ethics Quarterly, 17(4), 743-749.

Hodgson, G. M. (2017). Karl Polanyi on economy and society: a critical analysis of core concepts. Review of Social Economy, 75(1), 1-25.

Jácome, H. (2016). Avances y desafios de la Economía Popular y Solidaria en el Ecuador. En S. d. Solidaria, Economía Solidaria. Historias y prácticas de su fortalecimiento. Quito, 131.

Kantis, H., Ishida, M. y Komori, M. (2002): Empresarialidad en economías emergentes: Creación y desarrollo de nuevas empresas en América Latina y el Este de Asia. Washington, D. C.: Banco Interamericano de Desarrollo.

Ley Orgánica de Economía Popular y Solidaria (2014). Superintendencia de Economía Popular y Solidaria. Obtenido en red en el enlace: file://C:/Users/USER/AppData/Local/Temp/Ley\%20Orgánica\%20de\%20Economía\%20Popular $\% 20 \mathrm{y} \% 20$ Solidaria.pdf 
Martin, R. L., \& Osberg, S. (2007). Social entrepreneurship: The case for definition. Stanford Social Innovation Review, Spring, 28-39.

MIES. (2009). Marco conceptual y lineamientos de acción para el Instituto de Economía Popular y Solidaria. Quito.

Minniti, M., Bygrave, W.D. y Autio, E. (2006): Global Entrepreneurship Monitor-Executive Report 2005. Babson Park, MA. London: Babson Collage y London Business School.

Molina, E. C., \& Córdova, J. D. (2014). El crédito de desarrollo humano asociativo en la economía social y solidaria de la provincia de Tungurahua-Ecuador. Analítika: revista de análisis estadístico, (7), 33-47.

Moreira, P., \& Urriolagoitia, L. (2011). Emprendimiento Social. Revista Española del Tercer Sector, (17), 17-40.

Mukesh, S., VanSandt, C. \& Baugous, A. (2009). Social Entrepreneurship: The Role of Institutions. Journal of Business Ethics. 85, 201-216.

Orrego, C. (2009). La fenomenología y el emprendimiento. Pensamiento y gestión, 27, 235-252.

Padilla-Martínez, M. P., Quispe-Otacoma, A. L., Nogueira-Rivera, D., \& Hernández-Nariño, A. (2016). Diagnóstico y perspectivas de fomento del emprendimiento como instrumento de desarrollo. Ingeniería Industrial, 37(1), 91-103.

Palacios Núñez, G. (2010). Emprendimientos sociales: integrado a los excluidos en el ámbito rural. Revista de Ciencias Sociales, 579.

Ramírez, M., Bernal, M., \& Fuentes, R. (2013). Emprendimiento y Desarrollo Manufactureros en las Entidades Federativas de México. Revista Problemas del Desarrollo, 167.

Salinas, F., \& Osorio, L. (2012). Emprendimiento y economía social, oportunidades y efectos en una sociedad en transformación. CIRIEC-España, Revista de Economía Pública, Social y Cooperativa, (75).

Santos, F. M. (2012). A positive theory of social entrepreneurship. Journal of Business Ethics, 111(3), 335-351. 
Santos, F., Barroso, M., \& Guzmán, C. (2013). La economía global y los emprendimientos sociales. Revista de la Economía Mundial, 35.

Schwartz, S. H. (2005). Robustness and fruitfulness of a theory of universals in individual values. Valores e trabalho, 56-85.

Seelos, C., \& Mair, J. (2005). Social entrepreneurship: Creating new business models to serve the poor. Business Horizons, 48, 241-246. 